\title{
Individuals at the origin in the critical catalytic branching random walk
}

\author{
Valentin Topchii ${ }^{1 \dagger}$ and Vladimir Vatutin ${ }^{2 \ddagger}$ \\ ${ }^{1}$ Omsk Branch of Sobolev Institute of Mathematics, Pevtsov Street, 13, 644099 Omsk, Russia \\ ${ }^{2}$ Steklov Mathematical Institute, Gubkin Street, 8, 119991, Moscow, Russia \\ topchijeitam.omsk.net.ru, vatutinemi.ras.ru
}

A continuous time branching random walk on the lattice $\mathbb{Z}$ is considered in which individuals may produce children at the origin only. Assuming that the underlying random walk is symmetric and the offspring reproduction law is critical we prove a conditional limit theorem for the number of individuals at the origin.

Keywords: catalytic branching random walk; critical two-dimensional Bellman-Harris process

\section{Statement of problem and main results}

We consider the following modification of a standard branching random walk on $\mathbb{Z}$. Consider a population of individuals evolving as follows. The population is initiated at time $t=0$ by a single particle. Being outside the origin the particle performs a continuous time random walk on $\mathbb{Z}$ with infinitesimal transition matrix

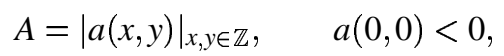

until the moment when it hits the origin. At the origin it spends an exponentially distributed time with parameter 1 and then either jumps to a point $y \neq 0$ with probability $-(1-\alpha) a(0, y) a^{-1}(0,0)$ or dies with probability $\alpha$ producing just before the death a random number of children $\xi$ in accordance with offspring generating function

$$
f(s) \stackrel{\text { def }}{=} \mathbb{E} s^{\xi}=\sum_{k=0}^{\infty} f_{k} s^{k} .
$$

At the birth moment the newborn particles are located at the origin but after this moment behave independently and stochastically the same as the parent individual.

The model we consider here is a particular case of the so-called branching random walk in catalytic medium. The longtime behavior of branching random walks in catalytic media of various types were investigated by a number of authors (see cf. (GKW99) and (W91) and the relevant references therein).

\footnotetext{
${ }^{\dagger}$ Supported by grants: RFBR 03-01-00045, INTAS-00-0265, Russian Scientifi c School - 2139.2003.1

${ }^{\ddagger}$ Supported by the grants: RFBR 02-01-00266, Russian Scientifi c School - 1758.2003.1 
Initially such models where investigated under the assumption that the initial population is infinite and generated by a stochastic field in $\mathbb{R}^{d}$ and the catalytic field may be random and infinite. Another situation was considered in (AB00), (ABY98), (BY98m) and (BY98d) where the authors studied some basic properties of a branching random walk in which the initial population was finite as well as the number of catalytic points.

Here we continue to investigate the last model and restrict ourselves by considering only one catalytic points. This model admits the following interpretation in terms of a queueing system with random number of working channels. The individuals in the population are identified with channels. The distant of a particle from the origin is considered as the queue length to the respective channel. The number of individuals outside the origin is the number of busy channels while the number of free channels. A channel having no customers after exponential time either becomes busy or produces a random number of new channels or becomes broken forever. The reader can find a more detailed description of this interpretation in (TVY03).

We impose the following restrictions on the characteristics of the process:

Hypothesis (I): The underlying random walk on $\mathbb{Z}$ is symmetric, irreducible and homogeneous $(a(x, y)=$ $a(0, y-x) \stackrel{\text { def }}{=} a(y-x)$ ) with $a(x) \geq 0$ as $x \neq 0, a(0)<0$,

$$
\sum_{x \in \mathbb{Z}} a(x)=0 \quad \text { and } \quad b^{2} \stackrel{\text { def }}{=} \sum_{x \in \mathbb{Z}} x^{2} a(x)<\infty .
$$

Hypothesis (II): The offspring process is critical $\left(f^{\prime}(1)=1\right)$ and $\sigma^{2} \stackrel{\text { def }}{=} f^{\prime \prime}(1) \in(0, \infty)$.

Let $\zeta(t)$ denote the number of particles in the process at time $t$ at the origin, $\mu(t)$ denote the number of particles in the process at time $t$ outside the origin, and let $\eta(t)=\zeta(t)+\mu(t)$ be the total number of individuals at the process at time $t$.

In (AB00), (ABY98), (BY98m), (BY98d) the long-time behavior of the expectations of $\eta(t), \zeta(t)$ and $\mu(t)$ was investigated. More delicate properties of the processes were studied in (TVY03). In particular, the following theorems were established in the mentioned paper.

Denote

$$
K \stackrel{\text { def }}{=} \frac{2^{3 / 4}}{\sigma \pi^{1 / 4}} \sqrt{\frac{b(1-\alpha)}{\alpha}}
$$

Theorem 1 (TVY03) Let hypotheses (I) and (II) be valid. Then

$$
\mathbb{P}(\mu(t)>0) \sim K t^{-1 / 4}, \quad t \rightarrow \infty,
$$

and for any $s \in[0,1]$

$$
\lim _{t \rightarrow \infty} \mathbb{E}\left[s^{\mu(t)} \mid \eta(t)>0\right]=1-\sqrt{1-s}
$$

Theorem 2 (TVY03) Under the conditions of Theorem 1, one has $\mathbb{P}(\eta(t)>0) \sim K t^{-1 / 4} \quad(t \rightarrow \infty)$; and for any $s \in[0,1]$, one has $\lim _{t \rightarrow \infty} \mathbb{E}\left[s^{\eta(t)} \mid \eta(t)>0\right]=1-\sqrt{1-s}$.

It follows from Theorems 1 and 2 that given $\{\eta(t)>0\}$ the probability is negligible that there are individuals at the origin. However, the changes in the size of the population may occur only when a particle hits the origin! For this reason to get a deeper insight into the evolution of $\eta(t)$ it is natural to pose the question on the properties of $\zeta(t)$. A particular answer to this question was obtained in (TVY03). 
Theorem 3 (TVY03) Under the conditions of Theorem 1

$$
q(t) \stackrel{\text { def }}{=} \mathbb{P}(\zeta(t)>0) \sim \frac{c_{q}}{\sqrt{t} \ln t}, \quad t \rightarrow \infty, \quad \text { where } \quad c_{q} \stackrel{\text { def }}{=} \pi K^{2}=\frac{2 \sqrt{2 \pi} b(1-\alpha)}{\sigma^{2} \alpha}
$$

In the present paper we continue to investigate properties of $\zeta(t)$ and prove the following conditional limit theorem.

Theorem 4 Let hypotheses (I) and (II) be valid. Then for any $\lambda \in[0, \infty)$

$$
\lim _{t \rightarrow \infty} \mathbb{E}\left\{\exp \left\{-\frac{\lambda \zeta(t)}{\mathbb{E}\{\zeta(t) \mid \zeta(t)>0\}}\right\} \mid \zeta(t)>0\right\}=\frac{1}{\lambda+1} .
$$

Theorem 4 is in the spirit of the standard conditional limit theorems for various types of the critical branching processes (Galton-Watson, age-dependent or general) (see, for instance (AN72)). However, our theorem has quite different nature. Indeed, the process at the origin may be viewed as a process with "reemigration" since any particle which left the origin returns to this point with probability 1 . Later on we give another interpretation of this process.

Before passing to the proof of Theorem 4 we say a few words about the asymptotic behavior of the scaling function $\mathbb{E}\{\zeta(t) \mid \zeta(t)>0\}$. To this aim we temporarily forget that our random walk has a point of catalyst and consider an ordinary random walk on $\mathbb{Z}$ satisfying Hypothesis (I). Assume that the random walk starts at the origin at time $t=0$ and let $\tau_{1}$ be the time spent by the walking particle at the origin until it leaves the origin and let $\tau_{2}$ be the time spent by this particle outside the origin until the first return to the origin.

Set $G_{1}(t) \stackrel{\text { def }}{=} \mathbb{P}\left(\tau_{1} \leq t\right)=1-e^{-t}, G_{2}(t) \stackrel{\text { def }}{=} \mathbb{P}\left(\tau_{2} \leq t\right)$ and

$$
G_{3}(t) \stackrel{\text { def }}{=} \alpha G_{1}(t)+(1-\alpha) G_{1} * G_{2}(t)
$$

It is shown in (TVY03) that the function $P(t) \stackrel{\text { def }}{=} \mathbb{E} \zeta(t)$ has the representation

$$
P(t)=\left(1-G_{1}(\cdot)\right) * U(t)
$$

where

$$
U(t) \stackrel{\text { def }}{=} \sum_{k=0}^{\infty} G_{3}^{* k}(t)
$$

Moreover, the following statement is valid:

Lemma 5 (TVY03) $P(t)$ is a monotone decreasing function and

$$
P(t) \sim \frac{1}{b \sqrt{2 \pi}(1-\alpha)} t^{-1 / 2} \stackrel{\text { def }}{=} c_{p} t^{-1 / 2}, \quad t \rightarrow \infty .
$$

In addition, there exists a constant $C>0$ such that for all $t \geq 0: \quad\left|P^{\prime}(t)\right| \leq C(t+1)^{-3 / 2}$. 
Combining this result and Theorem 3 gives

$$
\mathbb{E}\{\zeta(t) \mid \zeta(t)>0\}=\frac{\mathbb{E} \zeta(t)}{\mathbb{P}\{\zeta(t)>0\}}=\frac{P(t)}{q(t)} \sim c^{*} \ln t, \quad t \rightarrow \infty
$$

where $c^{*} \stackrel{\text { def }}{=} \alpha \sigma^{2}(4 \pi)^{-1} b^{-2}(1-\alpha)^{-2}$. Hence, recalling Theorem 4 we get the following conclusion.

Corollary 6 Under the conditions of Theorem 4 given $\{\zeta(t)>0\}$ the sequence $\zeta(t) /\left(c^{*} \ln t\right)$ converges weakly to an exponentially distributed random variable with mean 1.

\section{Branching random walk and Bellman-Harris processes}

In this section we prove Theorem 4 by introducing an auxiliary Bellman-Harris branching process with two types of particles $\left(Z_{1}(t), Z_{2}(t)\right)$ (see (H63; S74; V86p)) where by $Z_{i}(t), i=1,2$ we denote the number of individuals of type $i$ in this process at time $t$. The method of reduction the study of the longtime behavior of various characteristics of a branching random walk in catalytic medium to the investigation of asymptotic behavior of the corresponding characteristics of a two-type Bellman-Harris branching process was first suggested in (TVY03). It occurs to be fruitful in the present context to establish Theorem 4. Let

$$
F_{i}\left(t ; s_{1}, s_{2}\right)=\mathbb{E}\left\{s_{1}^{Z_{1}(t)} s_{2}^{Z_{2}(t)} \mid Z_{i}(0)=1\right\}, \quad i=1,2
$$

be the probability generating functions of the number of individuals of both types given that the process is initiated at time zero by a single individual of type $i$.

We would like to study the following critical Bellman-Harris process with two types of individuals. A particle of the first type has life length distribution $G_{1}(t)=\mathbb{P}\left(\tau_{1} \leq t\right)=1-e^{-t}, t \geq 0$. Dying it produces offspring of two types in accordance with probability generating function $f_{1}\left(s_{1}, s_{2}\right)=\alpha f\left(s_{1}\right)+(1-\alpha) s_{2}$, that is it produces with probability $\alpha f_{k}$ exactly $k$ particles of the first type and with probability $1-\alpha$ exactly one particle of the second type (recall the definition of $f(s)$ in (1)). The life length distribution of a particle of the second type is $G_{2}(t)=\mathbb{P}\left(\tau_{2} \leq t\right)$ (that is coincides in distribution with the time spend outside the origin by the parent individual of the catalytic branching random walk under investigation until the first return to the origin provided that the initial individual is located at point 0 at time $t=0$ and it does not produce children during its first stay at 0 ). Dying a particle of the second type produces offspring in accordance with probability generating function $f_{2}\left(s_{1}, s_{2}\right)=s_{1}$, that is, it produces exactly one particle of the first type and nothing else.

This Bellman-Harris process is critical and indecomposable since the maximal in absolute value eigenvalue (the Perron root) of the mean matrix

$$
M \stackrel{\text { def }}{=}\left\|\frac{\partial f_{i}}{\partial s_{j}}(1,1)\right\|_{i, j=1,2}=\left(\begin{array}{cc}
\alpha & 1-\alpha \\
1 & 0
\end{array}\right)
$$

of the process equals 1 and all the elements of $M^{2}$ are positive.

It is not difficult to understand that for the process constructed in such a way

$$
\left(Z_{1}(t), Z_{2}(t)\right) \stackrel{\text { distr }}{=}(\zeta(t), \mu(t)) .
$$


The main object of this section is to investigate the asymptotic behavior of the distribution of $Z_{1}(t)$ normalized by $\ln t$ and given $\left\{Z_{1}(t)>0\right\}$ as $t \rightarrow \infty$.

To this aim recall that under our assumption about the form of $f_{i}\left(s_{1}, s_{2}\right), i=1,2$, we have (see (S74), Chapter VIII, $\S 1$, or (H63))

$$
F_{1}\left(t ; s_{1}, s_{2}\right)=s_{1}\left(1-G_{1}(t)\right)+\int_{0}^{t}\left(\alpha f\left(F_{1}\left(t-u ; s_{1}, s_{2}\right)\right)+(1-\alpha) F_{2}\left(t-u ; s_{1}, s_{2}\right)\right) d G_{1}(u)
$$

and

$$
F_{2}\left(t ; s_{1}, s_{2}\right)=s_{2}\left(1-G_{2}(t)\right)+\int_{0}^{t} F_{1}\left(t-u ; s_{1}, s_{2}\right) d G_{2}(u) .
$$

Using the second of these equalities in the first one we get

$$
\begin{aligned}
F_{1}\left(t ; s_{1}, s_{2}\right) & =s_{1}\left(1-G_{1}(t)\right)+s_{2}(1-\alpha)\left(1-G_{2}(\cdot)\right) * G_{1}(t) \\
& +\int_{0}^{t} \alpha f\left(F_{1}\left(t-u ; s_{1}, s_{2}\right)\right) d G_{1}(u)+\int_{0}^{t}(1-\alpha) F_{1}\left(t-u ; s_{1}, s_{2}\right) d\left(G_{1} * G_{2}(u)\right) .
\end{aligned}
$$

Setting $s_{1}=s, s_{2}=1$ and

$$
1-F_{1}(t ; s, 1)=q(t, s)
$$

gives

$$
q(t, s)=(1-s)\left(1-G_{1}(t)\right)+\int_{0}^{t} q(t-u, s) d G_{3}(u)-\alpha \int_{0}^{t} h(q(t-u, s)) d G_{1}(u)
$$

where $h(x)=f(1-x)-(1-x)$ and $G_{3}(t)$ is defined in (1). Solving the renewal equation (6) with respect to $q(t, s)$ and taking (2) and (3) into account we obtain

$$
q(t, s)=(1-s) P(t)-\alpha \int_{0}^{t} h(q(t-u, s)) d\left(G_{1} * U(u)\right) .
$$

Since $G_{1}(t)=1-e^{-t}$, it follows that $\left(G_{1} * U(t)\right)^{\prime}=\left(1-G_{1}(\cdot)\right) * U(t)=P(t)$, and (7) reduces to

$$
q(t, s)=(1-s) P(t)-\alpha \int_{0}^{t} h(q(t-u, s)) P(u) d u .
$$

Clearly, $q(t, 0)=\mathbb{P}(\zeta(t)>0)=q(t)$. Let $s(t)=s(t, \lambda) \stackrel{\text { def }}{=} \exp \left\{-\frac{\lambda}{\max (1, \ln t)}\right\}$.

Since the integral in (8) is positive and $1-e^{-x} \leq x$ for $x \geq 0$ we have for $t \geq e$

$$
q(u, s(t))=\mathbb{E}\left\{1-\exp \left\{-\frac{\lambda \zeta(u)}{\ln t}\right\}\right\} \leq P(u) \frac{\lambda}{\ln t} \sim c_{p} u^{-1 / 2} \frac{\lambda}{\ln t}, \quad u \rightarrow \infty .
$$

In view of Hypothesis (II) and the Taylor formula $h(x) \sim \frac{\sigma^{2}}{2} x^{2} \quad$ as $\quad x \rightarrow 0$.

The last along with (9) and (4) allows us to get the following estimates for

$$
I(t) \stackrel{\text { def }}{=} \int_{0}^{t} h(q(t-u, s(t))) P(u) d u, \quad s=s(t, \lambda),
$$


that are uniform in $\lambda$ from any bounded interval $0 \leq \lambda \leq \Lambda<\infty$ :

$$
\begin{aligned}
I(t) & =\int_{t / 2}^{t} h(q(t-u, s)) P(u) d u+\frac{\lambda^{2}}{\ln ^{2} t} O(P(t))=P(t) \int_{0}^{t / 2} h(q(u, s)) d u \\
& +\int_{0}^{t / 2} h(q(u, s))(P(t-u)-P(t)) d u+\frac{\lambda^{2}}{\ln ^{2} t} O(P(t)) .
\end{aligned}
$$

Applying now the intermediate value theorem in the form $P(t-u)-P(t)=P^{\prime}(\theta(t, u)) u, t-u \leq$ $\theta(t, u) \leq t$, and recalling Lemma 5 we have for $t \geq e$ :

$$
\begin{aligned}
I(t) & =P(t) \int_{0}^{t / 2} h(q(u, s)) d u+\int_{0}^{t / 2} h(q(u, s)) P^{\prime}(\theta(t, u)) u d u+\frac{\lambda^{2}}{\ln ^{2} t} O(P(t)) \\
& =P(t) \int_{0}^{t / 2} h(q(u, s)) d u+\frac{\lambda^{2}}{\ln ^{2} t} O(P(t))=P(t) \frac{\sigma^{2}}{2} \int_{\ln t}^{t} q^{2}(u, s) d u+\frac{\lambda^{2}}{\ln t} o(P(t)) .
\end{aligned}
$$

By Theorem 3 if $x \ln t \rightarrow \infty$ then

$$
t^{x} \ln ^{2} t=\frac{1}{x^{2}} t^{x} \ln ^{2} t^{x} \sim \frac{c_{q}^{2}}{x^{2} q^{2}\left(t^{x}\right)} \quad \text { or } \quad \ln t \sim \frac{c_{q}^{2}}{t^{x} x^{2} q^{2}\left(t^{x}\right) \ln t} .
$$

Using the substitution $u=t^{x}$ and setting $\psi(t)=\ln \ln t / \ln t$ we get as $t \rightarrow \infty$

$$
\begin{aligned}
\int_{\ln t}^{t} q^{2}(u, s) d u & =\ln t \int_{\psi(t)}^{1} t^{x} q^{2}\left(t^{x}, \exp \left\{-\frac{\lambda x}{\ln t^{x}}\right\}\right) d x \\
& =(1+o(1)) \frac{c_{q}^{2}}{\ln t} \int_{\psi(t)}^{1} \frac{q^{2}\left(t^{x}, \exp \left\{-\frac{\lambda x}{\ln t^{x}}\right\}\right)}{x^{2} q^{2}\left(t^{x}\right)} d x
\end{aligned}
$$

Relations (10), (11) and (4) allow us to write

$$
-\alpha I(t)=\lambda^{2} o(q(t))-\alpha P(t) \frac{\sigma^{2} c_{q}^{2}}{2 \ln t} \int_{\psi(t)}^{1} \frac{q^{2}\left(t^{x}, \exp \left\{-\frac{\lambda x}{\ln t^{x}}\right\}\right)}{x^{2} q^{2}\left(t^{x}\right)} d x .
$$

The multiplier of the integral in (12) equals

$$
\alpha P(t) \frac{\sigma^{2} c_{q}^{2}}{2 \ln t} \sim \alpha c_{p} \frac{\sigma^{2} c_{q}^{2}}{2 \sqrt{t} \ln t}=\frac{c_{q}}{\sqrt{t} \ln t} \frac{\alpha \sigma^{2} K^{2} \pi}{b \sqrt{2 \pi}(1-\alpha) 2} \sim q(t) .
$$

Using this relation and introducing the notation

$$
g(t, \lambda) \stackrel{\text { def }}{=} \frac{q(t, s(t))}{q(t) \lambda}
$$

we rewrite (8) as follows

$$
g(t, \lambda)=c^{*}(1+o(1))-\int_{\lambda \psi(t)}^{\lambda} g^{2}\left(t^{u / \lambda}, u\right) d u, \quad t \rightarrow \infty, \quad 0<\lambda \leq \Lambda,
$$


where (recall (5))

$$
c^{*}=\frac{c_{p}}{c_{q}}=\frac{\alpha \sigma^{2}}{4 \pi b^{2}(1-\alpha)^{2}} .
$$

Note that $\quad c^{*} \sim \frac{P(t)}{q(t) \ln t}, \quad t \rightarrow \infty, \quad$ and that $\quad 0<\sup _{t \geq 0,0<\lambda} g(t, \lambda) \stackrel{\text { def }}{=} c_{1}-1<\infty$

in view of positivity of $q(t, s(t))$ and estimates (5) and (9). Consider the integral equation

$$
g(\lambda)=c^{*}-\int_{0}^{\lambda} g^{2}(u) d u, \quad \lambda \geq 0 .
$$

It is not difficult to check that

$$
g(\lambda)=\frac{c^{*}}{1+c^{*} \lambda}
$$

is a unique solution of (15). Using this fact and (13) we see that for any $\varepsilon>0$ there exists $t_{0}$ such that for all $t \geq t_{0}$ and $0<\lambda \leq \Lambda$

$$
|g(t, \lambda)-g(\lambda)| \leq \varepsilon+\int_{\lambda \psi(t)}^{\lambda}\left|g^{2}\left(t^{y / \lambda}, y\right)-g^{2}(y)\right| d y \leq \varepsilon+c_{1} \int_{\lambda \psi(t)}^{\lambda}\left|g\left(t^{y / \lambda}, y\right)-g(y)\right| d y .
$$

Select now $\Lambda>0$ such that $c_{1} \Lambda<1$ and set

$$
\Delta(t)=\sup _{0 \leq u \leq \Lambda}|g(t, u)-g(u)| .
$$

Clearly, for $0 \leq \lambda \leq \Lambda$

$$
\begin{aligned}
|g(t, \lambda)-g(\lambda)| & \leq \varepsilon+c_{1} \lambda \sup _{\lambda \psi(t) \leq y \leq \lambda}\left|g\left(t^{y / \lambda}, y\right)-g(y)\right| \\
& \leq \varepsilon+c_{1} \lambda \sup _{v \geq \ln t} \sup _{0 \leq y \leq \lambda}|g(v, y)-g(y)| \\
& \leq \varepsilon+c_{1} \Lambda \sup _{v \geq \ln t} \Delta(v)
\end{aligned}
$$

and, therefore, $\Delta(t) \leq \varepsilon+c_{1} \Lambda \sup _{v \geq \ln t} \Delta(v)$. Hence, putting $\Delta_{T}=\sup _{t \geq T} \Delta(t)$ and $\Delta=\lim _{T \rightarrow \infty} \Delta_{T}$, we see that $\Delta_{T} \leq \varepsilon+c_{1} \Lambda \sup _{t \geq T} \sup _{v \geq \ln t} \Delta(v) \leq \varepsilon+c_{1} \Lambda \sup _{v \geq \ln T} \Delta(v)=\varepsilon+c_{1} \Lambda \Delta_{\ln T}$ and $\Delta \leq \varepsilon+c_{1} \Lambda \Delta$. Whence $\Delta=0$ in view of $c_{1} \Lambda<1$ and arbitrariness of $\varepsilon>0$. Thus, we have proved that

$$
\lim _{t \rightarrow \infty} g(t, \lambda)=g(\lambda)=\frac{c^{*}}{1+\lambda c^{*}}, \quad 0<\lambda \leq \Lambda,
$$

or, recalling the definition of $g(t, \lambda)$

$$
\lim _{t \rightarrow \infty} \mathbb{E}\left\{\exp \left\{-\frac{\lambda \zeta(t)}{\ln t}\right\} \mid \zeta(t)>0\right\}=1-\lambda \lim _{t \rightarrow \infty} g(t, \lambda)=\frac{1}{1+\lambda c^{*}}
$$


for $0 \leq \lambda \leq \Lambda$.

Since the Laplace transform of a nonnegative random variable is an analytic and bounded function in the domain $\Re \lambda>0$ and the same is true for the function $1 /\left(1+\lambda c^{*}\right)$, it follows from the uniqueness theorem for analytic functions that (16) is valid for all $\lambda$ with $\Re \lambda>0$.

Now (16) and (14) show that

$$
\lim _{t \rightarrow \infty} \mathbb{E}\left\{\exp \left\{-\frac{\lambda \zeta(t) q(t)}{P(t)}\right\} \mid \zeta(t)>0\right\}=\frac{1}{1+\lambda}, \quad \lambda \geq 0 .
$$

The last and (5) complete the proof of Theorem 4.

\section{References}

[AB00] S. Albeverio, and L. V. Bogachev (2000) Branching random walk in a catalytic medium. I. Basic equations, Positivity, 4, No 1, 41-100.

[ABY98] S. Albeverio, L. V. Bogachev, and E. B. Yarovaya (1998) Asymptotics of branching symmetric random walk on the lattice with a single source, C.-R.-Acad.-Sci.-Paris-Ser--I-Math., 326, No 8, 975-980.

[AN72] K. B. Athreya, and P.E. Ney (1972) Branching processes, Springer-Verlag, New York-Heidelberg.

[BY98m] L. V. Bogachev, and E. B. Yarovaya (1998) A limit theorem for a supercritical branching random walk on $\mathbb{Z}^{d}$ with a single source, Russian-Math.-Surveys, 53, No 5, 1086-1088.

[BY98d] L. V. Bogachev, and E. B. Yarovaya (1998) Moment analysis of a branching random walk on a lattice with a single source, Dokl.-Akad.-Nauk, 363, No 4, 439-442 (in Russian).

[GKW99] A. Greven, A. Klenke, A. Wakolbinger. (1999) The longtime behavior of branching random walk in a catalytic medium. Electron. J. Probab. 4, no. 12, 80 pp. (electronic).

[H63] T.E.Harris (1963) The Theory of Branching Processes, Springer-Verlag, Berlin-GöttingenHeidelberg.

[S74] B. A. Sewastjanow (1974) Verzweigungsprozesse. Akademie-Verlag, Berlin.

[TVY03] V. A. Topchii, V. A. Vatutin, and E. B. Yarovaya (2003) Catalytic branching random walk and queueing systems with random number of independent servers, Theory of Probability and Mathematical Statistics, 69. (In print.)

[V86p] V. A. Vatutin (1979) Discrete limit distributions of the number of particles in a Bellman-Harris branching process with several types of particles, Theory Probab. Appl., 24, 509-520.

[V86m] V. A. Vatutin (1986) Critical Bellman-Harris branching processes starting with a large number of particles, Mat.-Zametki, 40, No. 4, 527-541.

[W91] A. Wakolbinger (1991) On the structure of entrance laws in discrete spatial critical branching processes, Math. Nachr. $151,51-57$. 